\title{
Solidificação direcional do eutético $\mathrm{Al}_{2} \mathrm{O}_{3} / \mathrm{GdAlO}_{3}$ por fusão a laser (Directional solidification of the $\mathrm{Al}_{2} \mathrm{O}_{3} / \mathrm{GdAlO}_{3}$ eutectic by laser melting technique)
}

\author{
E. R. M. Andreeta ${ }^{\prime}$, J. A. Rodrigues ${ }^{2}$, M. R. B. Andreeta ${ }^{I}$, F. Agulló-Rueda ${ }^{3}$, A. C. Hernandes ${ }^{1}$. \\ ${ }^{1}$ Centro Multidisciplinar para o Desenvolvimento de Materiais Cerâmicos \\ Grupo Crescimento de Cristais e Materiais Cerâmicos - IFSC \\ Universidade de S. Paulo, C.P. 369, 135670-970, S. Carlos, SP, Brasil. \\ ${ }^{2}$ Departamento de Engenharia de Materiais \\ Universidade Federal de S. Carlos, Brasil \\ ${ }^{3}$ Consejo Superior de Investigaciones Cientificas, Madrid, Espanha. \\ hernandes@if.sc.usp.br
}

\begin{abstract}
Resumo
Fibras eutéticas de $\mathrm{Al}_{2} \mathrm{O}_{3} / \mathrm{GdAlO}_{3}$ livres de poros foram produzidas empregando-se a técnica de fusão a laser denominada Laser Heated Pedestal Growth - LHPG. A microestrutura analisada em microscópio eletrônico de varredura foi denominada como do tipo "escrita chinesa", com a presença de padrões fibrosos em algumas amostras. Resultados de espectroscopia microRaman possibilitaram indexar as fases presentes e revelaram uma maior continuidade da fase $\mathrm{GdAlO}_{3}$ fibrosa, em comparação à "escrita chinesa”. Ensaios de flexão a três pontos resultaram em valores de módulo de ruptura no intervalo de 490 MPa a 1316 MPa.

Palavras-chave: Solidificação, Eutético, Fibra
\end{abstract}

\begin{abstract}
Pore-free eutectic fibers of $\mathrm{Al}_{2} \mathrm{O}_{3} / \mathrm{GdAlO}_{3}$ were produced by the laser melting technique named LHPG. The "chinese script" microstructure was observed using scanning electron microscopy. Fibrous pattern could be found in some of the samples. MicroRaman spectroscopy could index the eutectic phases and showed the continuity of the fibrous $\mathrm{GdAlO}_{3}$ when compared to the “chinese script" pattern. Three points bending measurements resulted in values of modulus of rupture varying from $490 \mathrm{MPa}$ to $1316 \mathrm{MPa}$.
\end{abstract}

Keywords: Solidification, Eutectic, Fiber.

\section{INTRODUÇÃO}

A solidificação direcional de ligas eutéticas pode ser usada como um meio de produção de compósitos livres de porosidade diretamente a partir da fase líquida. Dos materiais eutéticos, os compostos óxidos começaram a ser pesquisados mais efetivamente no final da década de 60 e início de 70 [1]. Esses materiais eutéticos eram em sua maioria a base de óxido de alumínio, tais como $\mathrm{Al}_{2} \mathrm{O}_{3} / \mathrm{Y}_{3} \mathrm{Al}_{5} \mathrm{O}_{12}, \mathrm{Al}_{2} \mathrm{O}_{3} / \mathrm{ZrO}_{3}$, $\mathrm{Al}_{2} \mathrm{O}_{3} / \mathrm{ZrO}_{2} / \mathrm{Y}_{2} \mathrm{O}_{3}, \mathrm{Al}_{2} \mathrm{O}_{3} / \mathrm{ZrO}_{2} / \mathrm{CaO}$ devido as suas boas propriedades mecânicas a temperaturas elevadas [1-3].

O eutético $\mathrm{Al}_{2} \mathrm{O}_{3} / \mathrm{GdAlO}_{3}\left(77 \mathrm{~mol} \%\right.$ de $\mathrm{Al}_{2} \mathrm{O}_{3}$ e $23 \mathrm{~mol} \%$ de $\left.\mathrm{Gd}_{2} \mathrm{O}_{3}\right)$ foi obtido pela primeira vez em 1997. Policristais cilíndricos de dimensões $10 \mathrm{~mm}$ de diâmetro e $10 \mathrm{~mm}$ de altura foram preparados em cadinhos de molibdênio que foram transladados a uma taxa de $5 \mathrm{~mm} / \mathrm{h}$ [4]. A microestrutura dos policristais foi analisada no microscópio eletrônico de varredura (MEV) e denominada "escrita chinesa. Esse eutético possui várias aplicações na área de engenharia mecânica e espera-se que possa ser utilizado em revestimentos de estruturas de turbinas de gás e sistemas de geração de energia, devido à sua alta estabilidade mecânica até próximo de sua temperatura de fusão (1873 K) [4].

Mais recentemente (1999), foram produzidas fibras de $\mathrm{Al}_{2} \mathrm{O}_{3} / \mathrm{GdAlO}_{3}$ usando a técnica denominada de "micropulling-down" [5, 6]. Nesta técnica, a fase líquida é solidificada progressivamente por meio do puxamento do material fundido, contido em um cadinho de irídio, aquecido por rádio-freqüência. A microestrutura da seção transversal das fibras de $\mathrm{Al}_{2} \mathrm{O}_{3} / \mathrm{GdAlO}_{3}$ apresentou um padrão complexo, contendo "escrita chinesa" mais padrões fibrosos. Não se conseguiu obter somente um tipo de microestrutura nesse eutético.

Nesse trabalho são relatadas as condições experimentais para a solidificação direcional de fibras eutéticas de $\mathrm{Al}_{2} \mathrm{O}_{3} / \mathrm{GdAlO}_{3}$ usando a técnica de fusão a laser, denominada Laser Heated Pedestal Growth (LHPG). Entre as vantagens dessa técnica, o elevado gradiente de temperatura axial associado ao processo de solidificação é o mais importante, pois permite maiores taxas de puxamento. Usando dessa característica particular foram produzidos materiais eutéticos de $\mathrm{Al}_{2} \mathrm{O}_{3} /$ $\mathrm{GdAlO}_{3}$ no formato de fibras com microestrutura controlada, ao contrário das obtidas pela técnica "micropulling-down" [5, 6]. Foram usadas as técnicas de microscopia eletrônica de varredura (MEV), espectroscopia microRaman e ensaios de flexão a três pontos para caracterização dos eutéticos produzidos.

\section{EXPERIMENTAL}

A técnica de fusão a laser

O método empregado neste trabalho para a obtenção dos 


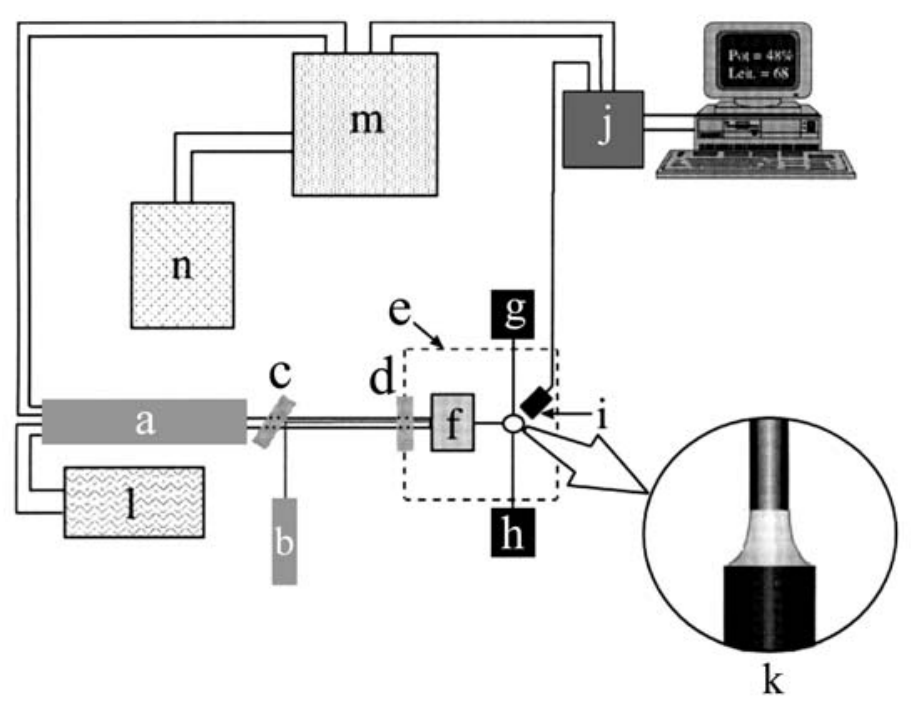

Figura 1: Representação esquemática dos principais componentes da técnica denominada LHPG $\boldsymbol{a}$ - Laser de $\mathrm{CO}_{2}, \boldsymbol{b}$ - Laser de He-Ne, $\boldsymbol{c}$ - Divisor de feixe de ZnSe, $\boldsymbol{d}$ - Janela de ZnSe, $\boldsymbol{e}$ - Câmara de puxamento, $\boldsymbol{f}$ - Sistema de focalização, $\boldsymbol{g}$ e $\boldsymbol{h}$ - respectivamente sistema de puxamento da fibra e elevação do pedestal, $\boldsymbol{i}$ - fototransistor, $\boldsymbol{j}$ - sistema de controle e estabilização da potência do laser, $\boldsymbol{k}$ - esquema da zona fundida, $\boldsymbol{l}$ - sistema de refrigeração do laser de $\mathrm{CO}_{2}, \boldsymbol{m}$ e $\boldsymbol{n}$ - fontes de RF e alimentação respectivamente. [Figure 1: Schematic diagram of the main components of LHPG technique. $\boldsymbol{a}-\mathrm{CO}_{2}$ laser $\boldsymbol{b}-\mathrm{He}-\mathrm{Ne}$ laser, $\boldsymbol{c}-\mathrm{ZnSe}$ beam divisor, $\boldsymbol{d}-\mathrm{ZnSe}$ optical window $\boldsymbol{e}$-Pulling camera $\boldsymbol{f}$-Focalization system $\boldsymbol{g}$ e $\boldsymbol{h}$-Fiber pulling and pedestal elevation systems, respectively $\boldsymbol{i}-$ Phototransistor $\boldsymbol{j}$-Power control of the $\mathrm{CO}_{2}$ laser $\boldsymbol{k}-$ Melting zone format $\boldsymbol{l}$-Cooling of the $\mathrm{CO}_{2}$ laser, $\boldsymbol{m}$ e $\boldsymbol{n}-R F$ and dc power, respectively.]

eutéticos difere de outros métodos de preparação, basicamente no sistema de aquecimento, em que um laser de $\mathrm{CO}_{2}$ (Synrad - $100 \mathrm{~W}$ nominal $\mathrm{CW}$ e $\lambda=10,6 \mu \mathrm{m}$ ) foi utilizado. O laser de $\mathrm{CO}_{2}$ é refrigerado a água durante toda a realização do experimento de puxamento por meio de um circulador que mantém a temperatura em $(19,0 \pm 0,5){ }^{\circ} \mathrm{C}$. É necessário manter a potência do laser estável, para evitar variações de temperatura na fase líquida. Um feixe de luz na região do visível, proveniente de um laser de He-Ne (Optoeletrônica, $1 \mathrm{~mW}$ nominal, $\lambda=632,8 \mathrm{~nm}$ ) é usado em paralelo ao feixe de luz do laser de $\mathrm{CO}_{2}$ para auxiliar no processo de visualização. Um diagrama esquemático da técnica de fusão a laser está ilustrado na Fig. 1. Todo o sistema de puxamento foi construído sobre uma mesa óptica com o propósito de se eliminar possíveis vibrações mecânicas que poderiam afetar a estabilidade do processo de solidificação. O sistema óptico está fixado dentro de uma câmara construída em aço inoxidável.

Os feixes dos laser utilizados penetram na câmara de puxamento através de uma janela de $\mathrm{ZnSe}$ e seguem direto para o sistema de focalização, Fig. 2. O feixe do laser incide no sistema de focalização que é constituído por um reflaxicon (dois cones de cobre polidos acoplados), um espelho plano com furo elíptico e um espelho esférico com furo circular ao seu centro. Ao incidir no reflaxicon, o feixe é convertido de um feixe cilíndrico para uma casca cilíndrica, de onde prossegue até encontrar o espelho plano situado a $45^{\circ} \mathrm{em}$ relação ao feixe, seguindo finalmente para o espelho esférico onde é então focalizado sobre o pedestal do eutético $\mathrm{Al}_{2} \mathrm{O}_{3} / \mathrm{GdAlO}_{3}$.

$\mathrm{Na}$ Fig. 3 apresentamos as principais etapas envolvidas no puxamento das fibras eutéticas. A primeira é o alinhamento mecânico da semente e do pedestal, seguida pelo aquecimento até

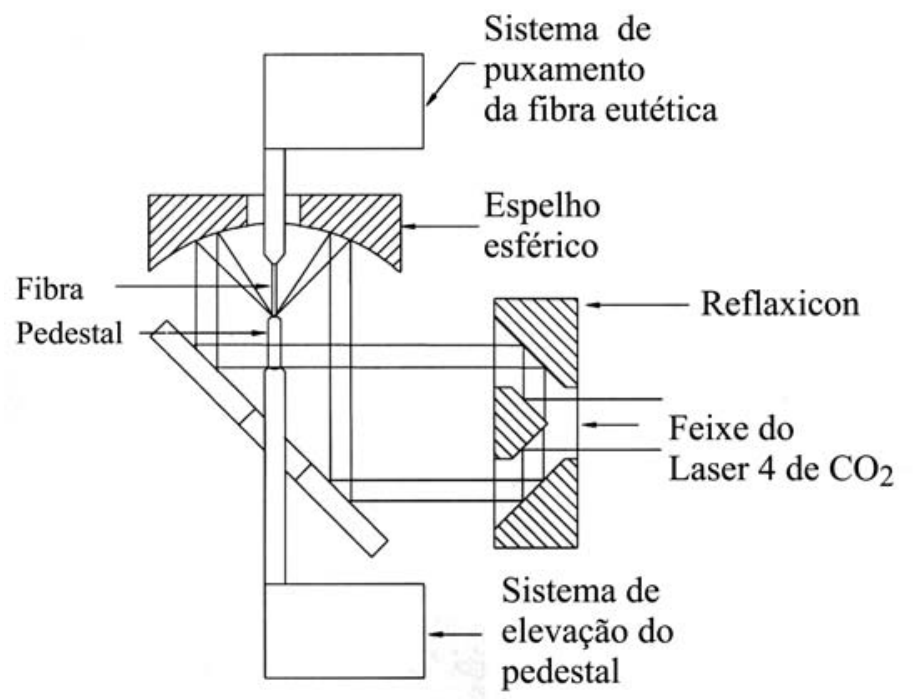

Figura 2: Representação esquemática do sistema de focalização usado para puxamento das fibras eutéticas.

[Figure 2: Schematic diagram of focalization system used for pulling eutectic fibers.]

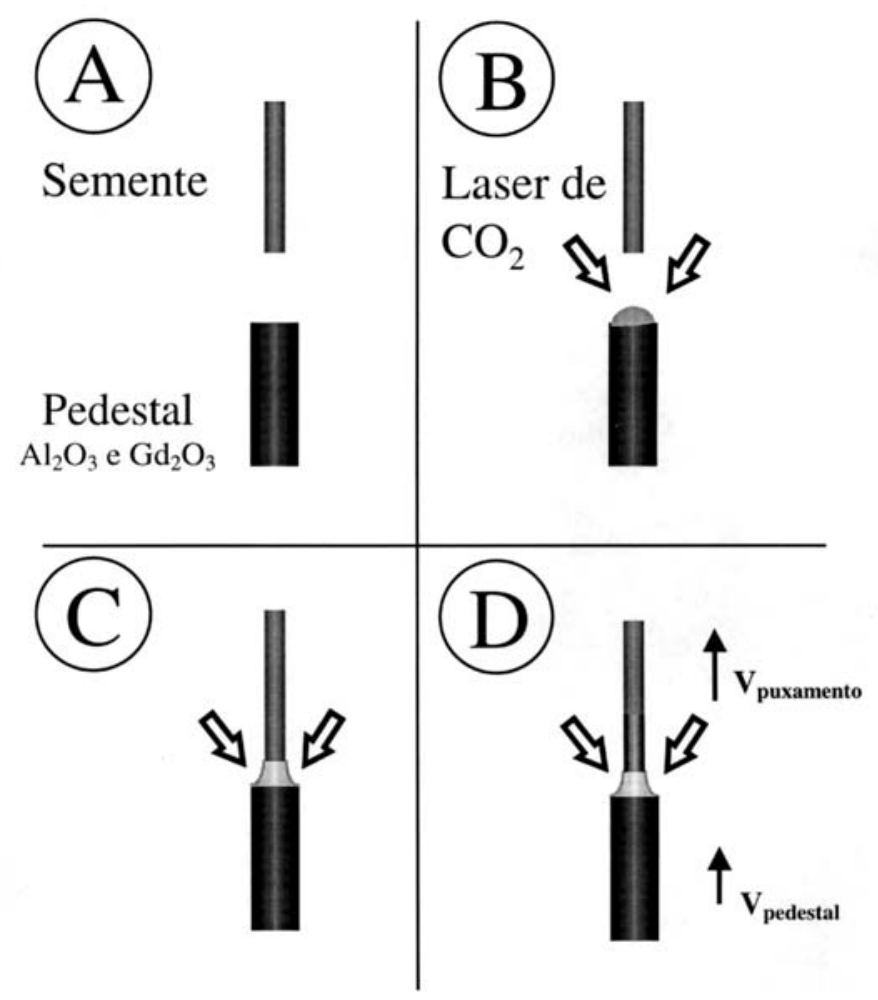

Figura 3: Etapas do processo de puxamento; A) Alinhamento mecânico entre semente e pedestal, B) Fusão do pedestal seguida do molhamento da semente no material fundido, C) Estabilização da temperatura e D) Início do processo de puxamento.

[Figure 3: Main steps of pulling process. A) Mechanical alignment between seed and pedestal B) Pedestal melting with contact of seed in liquid phase C) Temperature stabilization D) Start of pulling process of eutectic fibers.]

que uma pequena região fundida é formada, a semente então é levada em contato com a fase líquida. Após o equilíbrio termodinâmico ser atingido, inicia-se o processo de puxamento. Atingida a condição estável a semente e o pedestal são transladados simultaneamente, 
com taxas que podem ser iguais ou não, dependendo do diâmetro final da fibra eutética desejada.

\section{Preparação dos pedestais e fibras}

$\mathrm{O}$ pedestal usado no puxamento das fibras eutéticas de $\mathrm{Al}_{2} \mathrm{O}_{3} /$ $\mathrm{GdAlO}_{3}$ foi produzido pelo processo de extrusão a frio. A composição eutética extrudada foi de $77 \mathrm{~mol} \%$ de $\mathrm{Al}_{2} \mathrm{O}_{3}$ da Materials Research Co. e 23 mol\% de $\mathrm{Gd}_{2} \mathrm{O}_{3}$ da Reacton, ambos com grau de pureza $6 \mathrm{~N}$. Esses óxidos foram mecanicamente misturados em um almofariz de alumina, sendo depois ligados com a auxílio de uma solução de álcool polivinil (PVA). Essa mistura foi então compactada em um extrusor manual. Os pedestais resultantes possuíam 1 ou 2 mm de diâmetro e não precisaram ser processados, pois já apresentavam o formato cilíndrico necessário para uso em nosso equipamento. $\mathrm{O}$ uso desses pedestais sem sinterização permitiu realizar as três etapas da preparação do material (sintetização, sinterização e puxamento) diretamente no processo, quase que simultaneamente [7].

\section{Caracterização estrutural e mecânica}

Espectros microRaman foram obtidos para as diversas fibras de $\mathrm{Al}_{2} \mathrm{O}_{3} / \mathrm{GdAlO}_{3}$, usando um espectrômetro Raman Renishaw Ramascope 2000. Um laser com comprimento de onda igual a 632,8 $\mathrm{nm}$ foi utilizado como fonte de luz e focado sobre ambas as fases eutéticas com o auxílio de um microscópio óptico. Usamos um sistema de vídeo acoplado ao equipamento microRaman para visualização. Com um sistema de aquisição de dados acoplado a um microcomputador PC coletamos os espectros.

As amostras, tanto para as medidas Raman quanto para as análises de micrografia, consistiram de seções transversais e longitudinais de diversas fibras de $\mathrm{Al}_{2} \mathrm{O}_{3} / \mathrm{GdAlO}_{3}$ encapsuladas em resina epoxi, lapidadas e polidas com pasta de diamante, de granulação de $15 \mu \mathrm{m}$ até $1 \mu \mathrm{m}$.

Ensaios de flexão a três pontos foram realizados nas fibras eutéticas de aproximadamente $1 \mathrm{~mm}$ em diâmetro e $5 \mathrm{~cm}$ de comprimento para a obtenção do módulo de ruptura. $\mathrm{O}$ equipamento utilizado foi um MTS 810 com sensibilidade mínima de $20 \mathrm{~N}$.

\section{RESULTADOS E DISCUSSÃO}

Fibras eutéticas de $\mathrm{Al}_{2} \mathrm{O}_{3} / \mathrm{GdAlO}_{3}$, com dimensões variando entre $500 \mu \mathrm{m}$ a $1 \mathrm{~mm}$ de diâmetro e até $60 \mathrm{~mm}$ de comprimento, foram solidificadas unidirecionalmente pela técnica LHPG. A taxa de puxamento utilizada variou entre 4,2 e $60,0 \mathrm{~mm} / \mathrm{h}$. O gradiente de temperatura axial na interface sólido-líquido foi medido usando um pirômetro Minolta/Land, modelo Cyclops 152A e determinado como $(5,6 \pm 0,2) \times 10^{3} \mathrm{C} / \mathrm{cm}$. A principal dificuldade na preparação deste composto foi a presença de bolhas no líquido, que se formavam na interface pedestal-zona fundida, Fig. 4. Entretanto, verificou-se a presença de bolhas somente nas fibras puxadas com taxas maior ou igual a $48,0 \mathrm{~mm} / \mathrm{h}$.

A microestrutura eutética das fibras de $\mathrm{Al}_{2} \mathrm{O}_{3} / \mathrm{GdAlO}_{3}$ foi irregular e conhecida como "escrita chinesa", sendo típica deste eutético [4]. Entretanto, padrões fibrosos regulares juntamente com a "escrita chinesa" foram observados em algumas fibras. Um estudo correlacionando a microestrutura das fibras com a taxa de puxamento permitiu a determinação de um valor crítico, $24 \mathrm{~mm} / \mathrm{h}$,

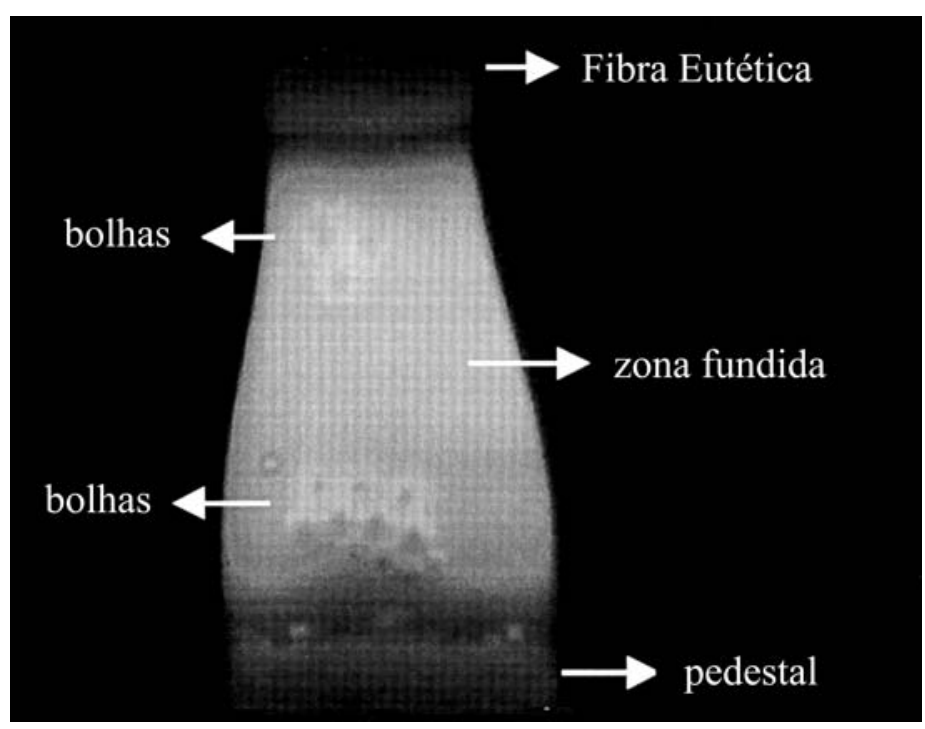

Figura 4: Bolhas presentes na zona fundida, durante um processo de puxamento de $\mathrm{Al}_{2} \mathrm{O}_{3} / \mathrm{GdAlO}_{3}$.

[Figure 4: Bubbles present in the melting zone during $\mathrm{Al}_{2} \mathrm{O}_{3} / \mathrm{GdAlO}_{3}$ pulling process.]
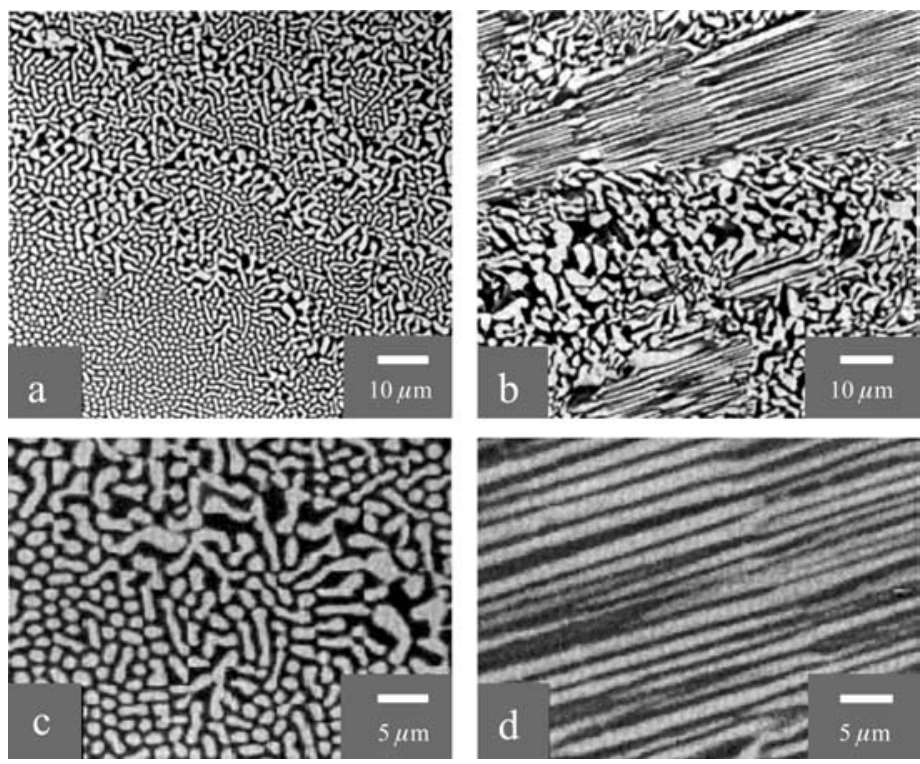

Figura 5: Microestrutura com arranjo complexo-regular ("escrita-chinesa" mais padrão fibroso) de fibras de $\mathrm{Al}_{2} \mathrm{O}_{3} / \mathrm{GdAlO}_{3}$. $\mathbf{a}$ - secção transversal de fibra puxada a $24 \mathrm{~mm} / \mathrm{h}$. b - ampliação do padrão complexo-regular da imagem a. c - secção longitudinal de fibra puxada a $42 \mathrm{~mm} / \mathrm{h}$, em que a continuidade da fibra é mais nítida. d - ampliação do padrão fibroso da imagem c.

[Figure 5: Microstructural patterns of $\mathrm{Al}_{2} \mathrm{O}_{3} / \mathrm{GdAlO}_{3}$ eutectic fibers with complex-regular arrangement ("Chinese script" and fibrous pattern). a - transversal section of a fiber pulled the $24 \mathrm{~mm} / \mathrm{h}$. $\boldsymbol{b}$-detail of complexregular arrangement showed in a.c-longitudinal section of a fiber pulled the $42 \mathrm{~mm} / \mathrm{h}$ showing the continuity in length $\boldsymbol{d}$-Detail of fibrous pattern showed in c.]

para a obtenção de somente microestrutura "escrita chinesa". $\mathrm{Na}$ Fig. 5, pode-se observar o arranjo "complexo-regular", que consiste em um padrão fibroso juntamente com a "escrita chinesa", presente nas fibras com diâmetro da ordem de $550 \mu \mathrm{m}$, e taxas de puxamento acima de $24 \mathrm{~mm} / \mathrm{h}$. A fase escura é o $\mathrm{Al}_{2} \mathrm{O}_{3}$ e a fase clara é o $\mathrm{GdAlO}_{3}$. As partes a e b ilustram o arranjo complexo-regular em seção transversal de um fibra com taxa de puxamento igual a $24 \mathrm{~mm} / \mathrm{h}$. 


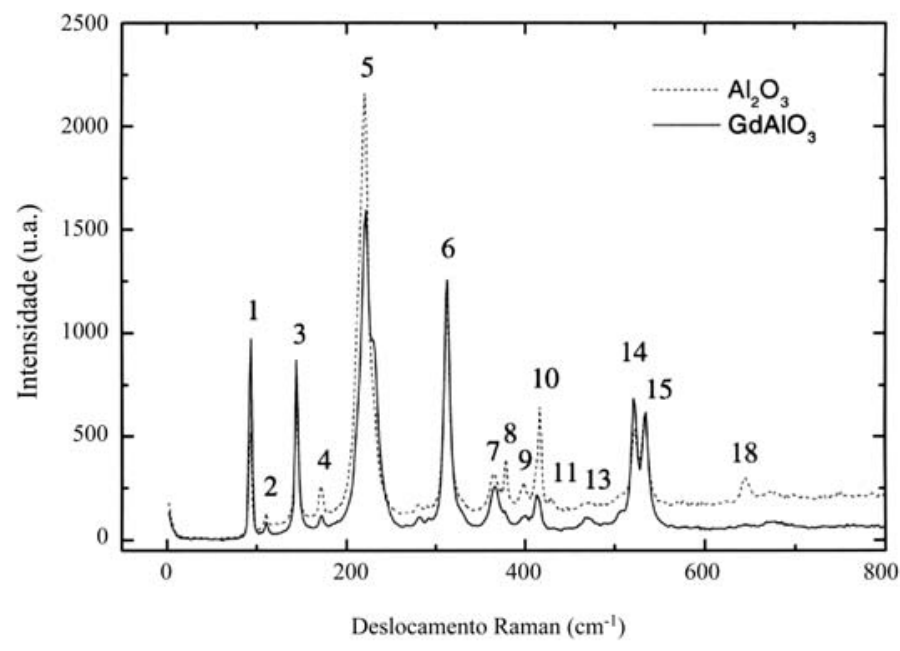

Figura 6: Espectros microRaman típicos obtidos para as fibras de $\mathrm{Al}_{2} \mathrm{O}_{3}$ / $\mathrm{GdAlO}_{3}$. A - Fase $\mathrm{GdAlO}_{3}, \mathrm{~B}-\mathrm{Fase} \mathrm{Al}_{2} \mathrm{O}_{3}$. Comprimento de onda incidente igual a $633 \mathrm{~nm}$, luz não polarizada.

[Figure 6: Typical micro-Raman spectra obtained for $\mathrm{Al}_{2} \mathrm{O}_{3} / \mathrm{GdAlO}$ fibers. $\mathrm{A}-\mathrm{GdAlO}_{3}$ phase $\mathrm{B}-\mathrm{Al}_{2} \mathrm{O}_{3}$ phase. Wavelength used was $633 \mathrm{~nm}$, not polarized light conditions.]

As partes c e d ilustram o padrão fibroso em seção longitudinal de uma fibra puxada a $42 \mathrm{~mm} / \mathrm{h}$, em que se evidencia a continuidade longitudinal da fase fibrosa. Foi reportado que arranjos regulares, como padrões fibrosos, podem surgir junto com a microestrutura irregular no caso da liga possuir nessa região um pequeno excesso do componente facetado [8]. Análises das frações volumétricas de ambas as fases nas fibras, por meio de suas micrografias, mostraram que as microestruturas com padrões fibrosos possuem um excesso de alumina, indicando que este é o componente facetado. Análises em fibras com diâmetro de $500 \mu \mathrm{m}$ a $640 \mu \mathrm{m}$ mostraram que a relação de Jackson-Hunt entre o espaçamento médio entre as fases eutéticas e a taxa de puxamento, $\lambda^{2} v=$ constante $=(6,3 \pm 0,1) \mu \mathrm{m}^{3} / \mathrm{s}$ foi obedecida em todo o intervalo de velocidades utilizado, permitindo o controle da microestrutura do $\mathrm{Al}_{2} \mathrm{O}_{3} / \mathrm{GdAlO}_{3}[8]$.

Espectros de microRaman obtidos para as fibras de $\mathrm{Al}_{2} \mathrm{O}_{3} /$ $\mathrm{GdAlO}_{3}$ estão ilustrados na Fig. 6, tanto para a fase $\mathrm{Al}_{2} \mathrm{O}_{3}$ quanto para o $\mathrm{GdAlO}_{3}$. Quando focado o laser na fase $\mathrm{Al}_{2} \mathrm{O}_{3}$, verificou-se a presença também dos picos do $\mathrm{GdAlO}_{3}$. Uma possível explicação para esse resultado pode ser relacionado a fraca intensidade dos deslocamentos Raman para a fase $\mathrm{Al}_{2} \mathrm{O}_{3}$ em comparação a da fase $\mathrm{GdAlO}_{3}$ lateral, prevalecendo a interação da luz laser na superfície no resultado final. Devemos notar, no entanto, que somente quando o laser foi focado na fase $\mathrm{Al}_{2} \mathrm{O}_{3}$ é que seus respectivos deslocamentos Raman são obtidos.

Na Tabela I estão as posições de todos os deslocamentos Raman para as fibras de $\mathrm{Al}_{2} \mathrm{O}_{3} / \mathrm{GdAlO}_{3}$. Os picos assinalados como 8, 11, 16 e 18 são correspondentes à $\alpha-\mathrm{Al}_{2} \mathrm{O}_{3}[9]$ e sempre se apresentaram com intensidade fraca nos espectros. O restante dos picos é referente ao $\mathrm{GdAlO}_{3}$ [10]. Apesar de uma distinção clara entre os espectros obtidos para as fases $\mathrm{Al}_{2} \mathrm{O}_{3}$ e $\mathrm{GdAlO}_{3}$ não ter ocorrido, medidas realizadas para amostras com maior tamanho das fases eutéticas (puxadas com menor velocidade) mostraram um aumento na intensidade dos picos 8 e 18 (referentes à alumina) quando o laser era focado na fase escura $\left(\mathrm{Al}_{2} \mathrm{O}_{3}\right)$.

Espectros de microRaman foram também obtidos focalizandose o laser em regiões com o padrão complexo-regular (região com
Tabela I - Posição dos picos dos espectros microRaman das fibras de $\mathrm{Al}_{2} \mathrm{O}_{3} / \mathrm{GdAlO}_{3}$.

[Table I-Position of the peaks of micro-Raman of $\mathrm{Al}_{2} \mathrm{O}_{3} / \mathrm{GdAlO}_{3}$ fibers.]

\begin{tabular}{lccc}
\hline $\mathbf{P I C}$ & POSIÇÃO $\left(\mathbf{c m}^{-1}\right)$ & PICO & POSIÇÃO $\left(\mathbf{c m}^{-1}\right)$ \\
\hline $\mathbf{1}$ & $93,0 \pm 0,2$ & $\mathbf{1 1}$ & $431 \pm 1$ \\
\hline $\mathbf{2}$ & $111,0 \pm 0,2$ & $\mathbf{1 2}$ & $449,0 \pm 0,2$ \\
\hline $\mathbf{3}$ & $146,0 \pm 0,2$ & $\mathbf{1 3}$ & $474,0 \pm 0,2$ \\
\hline $\mathbf{4}$ & $172,0 \pm 0,7$ & $\mathbf{1 4}$ & $521,0 \pm 0,2$ \\
\hline $\mathbf{5}$ & $222,0 \pm 0,8$ & $\mathbf{1 5}$ & $534,0 \pm 0,2$ \\
\hline $\mathbf{6}$ & $313,0 \pm 0,2$ & $\mathbf{1 6}$ & $567,0 \pm 0,2$ \\
\hline $\mathbf{7}$ & $366,0 \pm 0,2$ & $\mathbf{1 7}$ & $617,0 \pm 0,2$ \\
\hline $\mathbf{8}$ & $379,0 \pm 0,2$ & $\mathbf{1 8}$ & $646,0 \pm 0,2$ \\
\hline $\mathbf{9}$ & $399,0 \pm 0,2$ & $\mathbf{1 9}$ & $704,0 \pm 0,2$ \\
\hline $\mathbf{1 0}$ & $416 \pm 1$ & $\mathbf{2 0}$ & $741,0 \pm 0,2$ \\
\hline
\end{tabular}

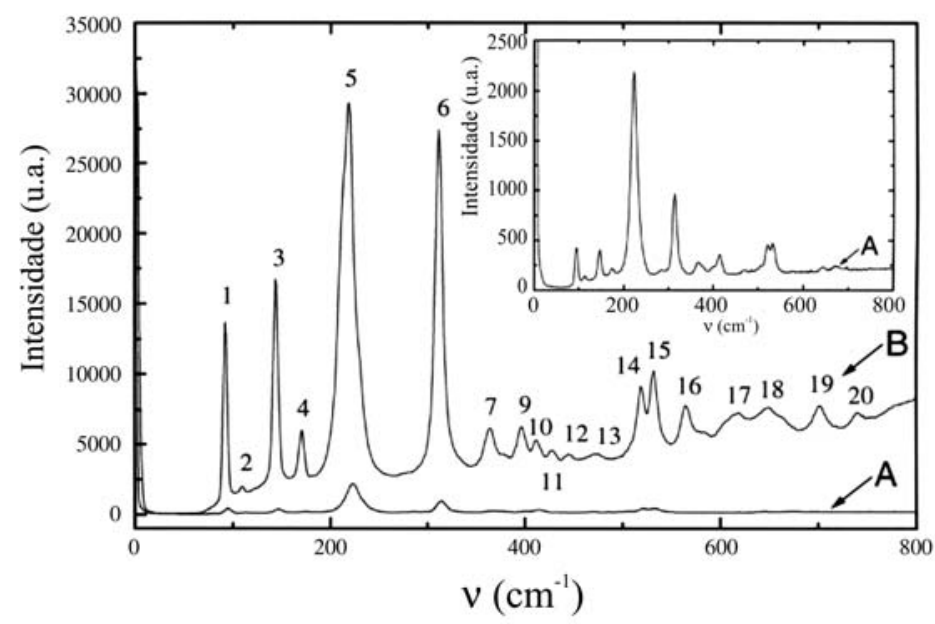

Figura 7: Espectro microRaman de fibra de $\mathrm{Al}_{2} \mathrm{O}_{3} / \mathrm{GdAlO}_{3}$. Laser de 633 nm usado na análise. A - Região com microestrutura "escrita chinesa". B - Região com padrão fibroso.

[Figure 7: $\mathrm{Al}_{2} \mathrm{O}_{3} / \mathrm{GdAlO}_{3}$ micro-Raman spectra. A-Region with "Chinese script" microstructure. B-Region with fibrous microstructure. Wavelength used was 633nm, not polarized light conditions.]

padrão fibroso). A comparação do espectro Raman para o padrão fibroso e o da microestrutura "escrita chinesa" está ilustrado na Fig. 7. Verificou-se que a intensidade Raman é muito maior no padrão fibroso, indicando que houve uma maior quantidade de material analisada no espectro B da Fig. 5. Isso é uma evidência de que há uma maior continuidade longitudinal da fase $\mathrm{GdAlO}_{3}$ nesse padrão fibroso do que na "escrita chinesa".

Na Tabela II apresentamos os valores médios dos módulos de ruptura (MOR) obtidos para as fibras de $\mathrm{Al}_{2} \mathrm{O}_{3} / \mathrm{GdAlO}_{3}$ com diâmetro aproximado de $1 \mathrm{~mm}$, puxadas com diferentes velocidades. Os valores determinados estão no intervalo de $490 \mathrm{MPa}$ a $1316 \mathrm{MPa}$, similares aos relatados para a alumina policristalina, que variam de 100 até $650 \mathrm{MPa}$, para distintos processamentos e diferentes tamanhos de 

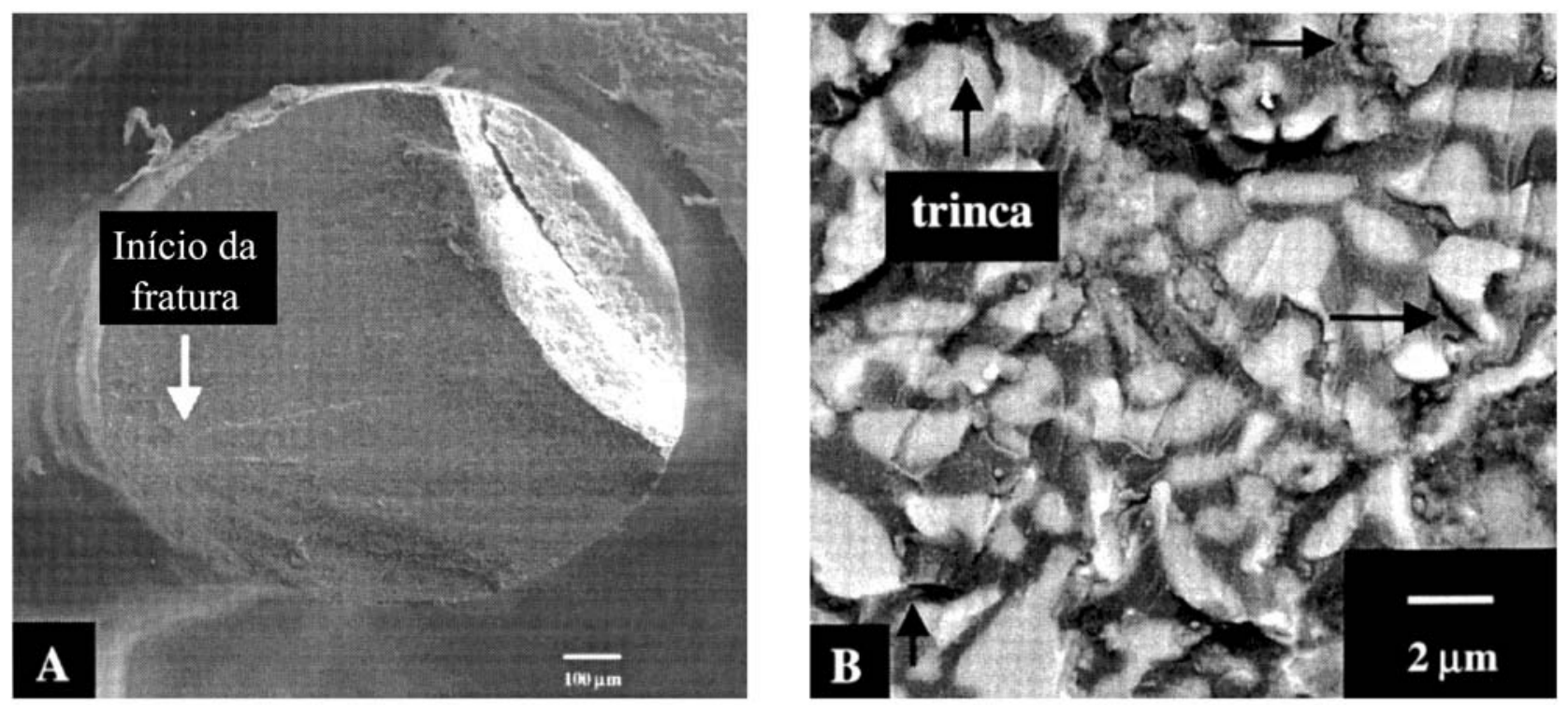

Figura 8: Superfície fraturada de fibra de $\mathrm{Al}_{2} \mathrm{O}_{3} / \mathrm{GdAlO}_{3}$. A - visão geral, pode-se notar o início da fratura. $\mathrm{B}-$ microestrutura da superfície fraturada, as setas indicam alguns destacamentos entre as fases e uma trinca.

[Figure 8: Fracture surface of $\mathrm{Al}_{2} \mathrm{O}_{3} / G d A l O_{3}$ fiber. A - Fiber showing the begin of the fracture. B - Fractured surface microstructure.]

Tabela II - Módulos de ruptura para fibras de $\mathrm{Al}_{2} \mathrm{O}_{3} / \mathrm{GdAlO}_{3}$. [Table II - Modulus of rupture of the $\mathrm{Al}_{2} \mathrm{O}_{3} / \mathrm{GdAlO}_{3}$ fibers.]

\begin{tabular}{cc}
\hline $\begin{array}{c}\text { Velocidade } \\
(\mathrm{mm} / \mathrm{h})\end{array}$ & $\begin{array}{l}\text { MOR } \\
(\mathrm{MPa})\end{array}$ \\
\hline $18^{*}$ & 730 \\
\hline $18^{*}$ & 574 \\
\hline 24 & 719 \\
\hline 30 & 507 \\
\hline 30 & 1316 \\
\hline 36 & 668 \\
\hline 36 & 943 \\
\hline 36 & 582 \\
\hline 48 & 759 \\
\hline 48 & 490 \\
\hline 60 & 1235 \\
\hline
\end{tabular}

grãos [11]. As regiões de fratura foram cuidadosamente observadas usando a técnica de microscopia eletrônica, Fig. 8. Nas superfícies fraturadas foi possível observar que as fibras sofreram fratura plana. Alguns destacamentos entre as fases estão presentes, porém com pequena ocorrência e em somente poucas regiões das superfícies, o que indica uma boa ligação entre as fases e a ausência de contornos de grão contendo fases amorfas.

\section{CONCLUSÕES}

Fibras óxidas eutéticas de $\mathrm{Al}_{2} \mathrm{O}_{3} / \mathrm{GdAlO}_{3}$ foram produzidas usando a técnica laser heated pedestal growth e a microestrutura obtida foi a "escrita chinesa" em fibras de aproximadamente $550 \mu \mathrm{m}$ puxadas a velocidades inferiores a $24 \mathrm{~mm} / \mathrm{h}$. Fibras com maiores taxas de puxamento apresentaram um padrão fibroso juntamente com a "escrita chinesa". As fibras consistem em cristais de $\mathrm{GdAlO}_{3}$ de boa qualidade envoltos em uma matriz de $\mathrm{Al}_{2} \mathrm{O}_{3}$. Ensaios mecânicos de flexão a três pontos resultaram em valores de módulo de ruptura para esses compósitos de $490 \mathrm{MPa}$ a $1316 \mathrm{MPa}$, demonstrando a boa resistência mecânica das fibras.

\section{AGRADECIMENTOS}

À FAPESP e ao CNPq pelo auxílio financeiro.

\section{REFERÊNCIAS}

[1] F. L. Kennard, R. C. Bradt, V. S. Stubican, J. Am. Ceram. Soc. 56, 11 (1973) 566.

[2] T. Lebeau, J. O. Strom-Olsen, J. E. Gruzleski, R. A. Drew, Mat. Charact. 35 (1995) 11.

[3] Y. Sung, I. Sung, J. Mat. Sci. 31 (1996) 4775.

[4] Y. Waku, N. Nakagawa, T. Wakamoto, H. Ohtsubo, K. Shimizu, Y. Kohtoku, Nature 389 (1997) 49.

[5] A. Yoshikawa, B. M. Epelbaum, K. Hasegawa, S. D. Durbin, T. Fukuda, J. Cryst. Growth 205 (1999) 305.

[6] A. Yoshikawa, K. Hasegawa, J. H. Lee, S. D. Durbin, B. M. Epelbaum, D. H. Yoon, T. Fukuda, Y. Waku, J. Cryst. Growth 218 (2000) 67.

[7] M. R. B. Andreeta, A. C. Hernandes, J. Cryst. Growth 200 (1999) 621. [8] E. R. M. Andreeta, M. R. B. Andreeta, A. C. Hernandes, J. Cryst. Growth 234 (2002) 782.

[9] H. Yao, H. Yan, J. Appl. Phys. 85, 9 (1999) 6717.

[10] V. S. Bagnato, Dissertação de Mestrado, IFSC/USP (1983).

[11] E. Dörre, H. Hübner, "Alumina: Processing, Properties, and Applications" Springer-Verlag, Berlin (1984) 329.

(Rec. 18/02/02, Ac. 28/02/02) 